\title{
NARROWING AND RESOLUTION IN LOGIC-FUNCTIONAL
}

\author{
PROGRAMMING LANGUAGES
}

\author{
Elio Giovannetti \\ Dipartimento di Informatica \\ Universita degli Studi di Torino \\ Corso Svizzera 185 \\ 10149 Torino, Italy
}

\section{(Abstract)}

The lecture will deal with the rôle of narrowing and/or resolution in the framework of integration between logic and functional programming.

Narrowing and flattening plus resolution are basically equivalent methods for performing semantical unification. The conditions under whoich this equivalence holds are carefully explained, and various "relative completeness" properties for narrowing-based and resolution-based semantic unification algorithms are derived, along with their application to the design and implementation of the logic-functional programming language K-LEAF. In addition, a sketch of the latest development of the K-LEAF/IDEAL logic-functional integration will be traced, especially for as concerns the implementation and the semantics of the higher-order component. 